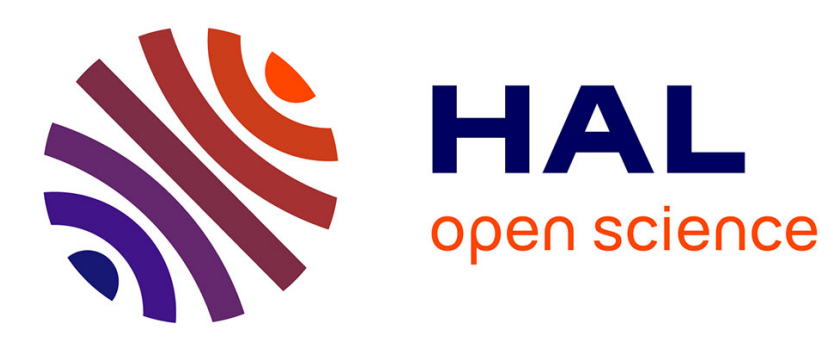

\title{
L'interprétation des discordances compétence-performance dans le développement cognitif. L'exemple de la quantification de l'inclusion Patrick Perret
}

\section{- To cite this version:}

Patrick Perret. L'interprétation des discordances compétence-performance dans le développement cognitif. L'exemple de la quantification de l'inclusion. Annee Psychologique, 2002, 102 (4), pp.693 723. 10.3406/psy.2002.29614 . hal-01779378

\section{HAL Id: hal-01779378 \\ https://hal.science/hal-01779378}

Submitted on 26 Apr 2018

HAL is a multi-disciplinary open access archive for the deposit and dissemination of scientific research documents, whether they are published or not. The documents may come from teaching and research institutions in France or abroad, or from public or private research centers.
L'archive ouverte pluridisciplinaire HAL, est destinée au dépôt et à la diffusion de documents scientifiques de niveau recherche, publiés ou non, émanant des établissements d'enseignement et de recherche français ou étrangers, des laboratoires publics ou privés. 


\section{L'interprétation des discordances compétence-performance dans le} développement cognitif. L'exemple de la quantification de l'inclusion Patrick Perret

\section{Citer ce document / Cite this document :}

Perret Patrick. L'interprétation des discordances compétence-performance dans le développement cognitif. L'exemple de la quantification de l'inclusion. In: L'année psychologique. 2002 vol. 102, n4. pp. 693-723;

doi : https://doi.org/10.3406/psy.2002.29614

https://www.persee.fr/doc/psy_0003-5033_2002_num_102_4_29614

Fichier pdf généré le 19/04/2018 


\begin{abstract}
Summary : Competence-performance discrepancies in cognitive development. The case of classinclusion reasoning

This paper explores, through the example of the class inclusion literature, the major hypotheses that have been proposed to account for competence-performance discrepancies in the course of cognitive development. It is shown that these hypotheses focused successively on each ofthe three parameters stated by Flavell and Wohlwill's initial model. A first set of empirical studies has established that performance varies as a function of task-related variables. A second set, making use of tasks with more complex logical demands, accounts for performance variability by a lack of logical competence that is supposed to emerge at adolescence. From a third perspective, competence is available early and discrepancies resuit from the inefficient inhibition of competing knowledge structures. After a discussion of the theoretical difficulties met by these alternative explanatory frameworks, we suggest that a « knowledge-levels » approach is necessary to coordinate the various hypotheses.

Key words : cognitive development, competence-performance, inhibition, knowledge-levels, class inclusion.
\end{abstract}

\title{
Résumé
}

Résumé

Cet article explore, à travers l'exemple de la quantification de l'inclusion, les principaux cadres théoriques avancés pour rendre compte des discordances compétence-performance au cours du développement cognitif. Leur recension critique indique que les hypothèses se sont successivement focalisées sur chacun des trois paramètres spécifiés dans le modèle initial de Flavell et Wohlwill (1969). Un premier courant de recherche a clairement démontré l'existence d'une variabilité intraindividuelle des performances en fonction des propriétés des épreuves utilisées. Un second, ayant recours à des épreuves aux exigences logiques accrues, rend compte de cette variabilité par un défaut de compétence logique, laquelle n'émergerait pas avant l'adolescence. Pour un troisième courant, la compétence logique serait à l'inverse précocement disponible, les discordances résultant d'un défaut d'inhibition de schèmes inadaptés. Après une discussion des difficultés théoriques rencontrées par ces hypothèses alternatives, nous suggérons qu'une approche en termes de "niveaux de connaissance » permettrait leur articulation.

Mots-clés : développement cognitif, compétence-performance, inhibition, niveaux de connaissance, quantification de l'inclusion. 
NOTES

Note théorique

Centre de Recherche en Psychologie de la Connaissance, du Langage et de l'Émotion, EA 3273

Université de Provence ${ }^{1}$

L'INTERPRÉTATION

DES DISCORDANCES COMPÉTENCE-PERFORMANCE DANS LE DÉVELOPPEMENT COGNITIF. L'EXEMPLE DE LA QUANTIFICATION DE L'INCLUSION

Patrick PerReT ${ }^{2} 3$

SUMMARY : Competence-performance discrepancies in cognitive development. The case of class-inclusion reasoning

This paper explores, through the example of the class inclusion literature, the major hypotheses that have been proposed to account for competenceperformance discrepancies in the course of cognitive development. It is shown that these hypotheses focused successively on each of the three parameters stated by Flavell and Wohlwill's initial model. A first set of empirical studies has established that performance varies as a function of task-related variables. A second set, making use of tasks with more complex logical demands, accounts for performance variability by a lack of logical competence that is supposed to emerge at adolescence. From a third perspective, competence is available early and discrepancies result from the inefficient inhibition of competing knowledge structures. After a discussion of the theoretical difficulties met by these alternative explanatory frameworks, we suggest that a "knowledge-levels" approach is necessary to coordinate the various hypotheses.

Key words : cognitive development, competence-performance, inhibition, knowledge-levels, class inclusion.

1. 29, avenue Robert-Schuman, 13621 Aix-en-Provence, Cedex 1.

2. E-mail : perretp@up.univ-aix.fr.

3. Je remercie vivement Jean-Louis Paour, Agnès Blaye et Christine Bailleux pour l'aide précieuse qu'ils ont apportée à la rédaction de ce texte. 
La psychologie du développement cognitif est de plus en plus fréquemment confrontée à des paradoxes développementaux. Ces paradoxes naissent de la mise à jour, par des moyens méthodologiques renouvelés, de compétences précoces dans divers domaines conceptuels, notamment ceux retenus par Piaget comme caractéristiques du développement logique chez l'enfant. La découverte de ces compétences précoces impose toutefois aux chercheurs de proposer de nouveaux cadres interprétatifs de la variabilité de la performance car les échecs tardifs aux épreuves opératoires n'ont, eux, jamais été démentis par les faits. L'enjeu théorique réside alors dans la signification accordée à cette variabilité intra-individuelle de la performance à des épreuves relevant pourtant du même domaine conceptuel. L'objectif de cet article est de faire le point sur les cadres interprétatifs actuellement disponibles pour rendre compte de cette variabilité, et d'en discuter les limites. Les nombreuses recherches menées sur le développement de la quantification de l'inclusion, particulièrement illustratives de cette problématique, seront utilisées pour soutenir cette discussion.

\section{L'ÉPREUVE DE QUANTIFICATION DE L'INCLUSION}

La position piagetienne concernant le développement de la compréhension du concept de classes en relation d'inclusion a été à plusieurs reprises discutée (voir notamment Bideaud, 1988). Nous nous contenterons donc ici d'en reprendre les principales propositions. L'épreuve de quantification de l'inclusion a d'abord été proposée dans le cadre des travaux de Piaget et Szeminska (1941-1967) sur la genèse du nombre, mais les étapes de sa réussite chez l'enfant ont été plus précisément étudiées dans les recherches menées avec Inhelder sur la genèse des structures logiques élémentaires (Piaget et Inhelder, 1959-1967). Elle consiste à présenter à l'enfant deux ensembles d'objets numériquement inégaux et correspondant à deux sous-classes $\left(\mathbf{A}>\mathbf{A}^{\prime}\right)$, elles-mêmes incluses dans une classe sur-ordonnée (B). La question dite de quantification consiste à demander à l'enfant : «Y a-t-il plus de $A$ ou plus de $B$ ? 》

Pour Piaget, la réussite à cette épreuve marque l'aboutissement de la notion de classe par coordination des définitions 
en intension et en extension. Sa résolution constitue l'un des marqueurs principaux de la nature opératoire de la pensée de l'enfant : «Il faut justement que l'enfant comprenne simultanément et la mobilité des parties, et la réversibilité des transformations et la conservation du tout $B$ au cours de ces transformations " (Piaget et Inhelder, 1959-1967, p. 109). Cette analyse des exigences opératoires de l'épreuve amène Piaget à formaliser la compétence cognitive nécessaire à sa résolution dans les termes d'une structure isomorphe au groupement additif des classes. D'un point de vue fonctionnel, la difficulté de l'épreuve consiste d'une part à envisager l'appartenance simultanée d'un objet à la sous-classe et à la classe sur-ordonnée, $d$ 'autre part à conserver le tout pendant qu'on lui compare une de ses parties.

Comme l'a montré Bideaud (1988), les travaux ultérieurs ont échoué à valider la prédiction d'une synchronie développementale entre la réussite à cette épreuve et à celle des autres épreuves opératoires, fragilisant ainsi l'hypothèse structurale. Par ailleurs, son lien avec l'étude du développement de la catégorisation s'est distendu sous l'influence de travaux révélant une relative indépendance entre les conduites de classification d'une part et les réponses des enfants aux questions de quantification d'autre part. Au total, la quantification de l'inclusion des classes s'est peu à peu constituée en champ de recherche autonome. L'évolution des performances à l'épreuve demeure un fait développemental majeur, au point que la plupart des auteurs postpiagetiens testeront la capacité de leur modèle théorique à en rendre compte, sans jamais parvenir à l'appréhender totalement. À en juger par la diversité des hypothèses théoriques convoquées, cette épreuve semble avoir peu à peu pris statut de paradigme (Howe, Rabinowitz et Powell, 1998). Elle constitue, aujourd'hui encore, un cadre privilégié pour l'étude du raisonnement logique et des phénomènes de discordances qui caractérisent son développement.

\section{LES DISCORDANCES COMPÉTENCE-PERFORMANCE}

L'essentiel des travaux anglo-saxons dans le domaine du raisonnement inclusif se sont fondés sur l'idée (plus ou moins explicite selon les auteurs) que l'échec à l'épreuve de quantification de l'inclusion ne relevait pas d'un déficit logique mais d'un déficit de performance dont l'origine serait à rechercher ailleurs que 
dans le développement des connaissances. Le coup d'envoi de ces recherches a été fourni par l'étude princeps de Wohlwill (1968) montrant que des modifications des conditions de présentation de l'épreuve pouvaient modifier les réponses successives d'un même enfant. Flavell et Wohlwill (1969) ont alors proposé de généraliser à l'étude du développement cognitif dans son ensemble la distinction, introduite par les psycholinguistes du courant chomskien, entre compétence et performance. La compétence correspond à la représentation formelle de la connaissance logique impliquée dans la résolution d'une épreuve. La performance renvoie, elle, à l'instanciation de cette connaissance lors de la résolution effective de l'épreuve. Des contraintes pèsent sur cette instanciation de sorte que la compétence du sujet pourra ou non s'exprimer dans sa performance en fonction des caractéristiques de la tâche. Pour Flavell et Wohlwill, la recherche des origines d'un échec doit faire la part entre deux registres d'interprétation : celui d'un défaut de compétence (le sujet échoue parce qu'il n'a pas encore élaboré la structure de connaissance impliquée) et celui d'une discordance compétenceperformance (le sujet échoue en raison de caractéristiques de l'épreuve qui interfèrent avec la mobilisation d'une compétence pourtant disponible). Le modèle proposé spécifie dès lors trois paramètres qui déterminent conjointement l'évolution des performances au cours du développement :

- disponibilité de la compétence. Le premier facteur impliqué dans la détermination de la performance renvoie bien sûr à la formation de la connaissance logique qui sous-tend la résolution de l'épreuve. Mais il ne peut, à lui seul, rendre compte de la variabilité de la performance comme en attestent, selon les auteurs, les phénomènes de décalages horizontaux observés par Piaget lui-même ;

- facteur tâche. Il correspond aux caractéristiques de l'épreuve utilisée pour évaluer la compétence. Ses propriétés (familiarité du matériel, présentation de l'information pertinente, taux d'informations interférentes, charge mnésique...) modulent la probabilité que la compétence se révèle dans la performance ;

- sensibilité du sujet au facteur tâche. L'influence des propriétés de l'épreuve sur la performance varie elle-même avec l'âge et ce troisième facteur vient par conséquent moduler l'influence du second. 
Pour Flavell et Wohlwill, la compréhension des discordances compétence-performance, de leur émergence et de leur disparition, impliquait de prendre simultanément en compte le poids relatif de chacun de ces paramètres au cours du développement. Cette proposition a toutefois été assez largement ignorée au profit d'une focalisation successive sur chacun des trois facteurs. Un premier courant de recherche s'est exclusivement consacré à l'identification des propriétés situationnelles susceptibles de faire varier les réponses des enfants.

\section{LE RÔLE DU FACTEUR TÂCHE}

\section{DANS LA DÉTERMINATION DE LA PERFORMANCE}

Pascual-Leone et Bovet (1966) ont qualifié l'épreuve d'inclusion de «situation déroutante » en ce que sa résolution implique non seulement de coordonner les informations pertinentes mais également de résister à des schèmes intuitifs non pertinents que le problème tend à activer. Il est clair pour ces auteurs que la propriété déroutante de l'épreuve (comme celle de toutes les autres épreuves opératoires) est une intention délibérée de Piaget de tester ainsi la solidité de la connaissance logique en construction. Pour la plupart des auteurs anglosaxons en revanche, cette caractéristique constitue un obstacle à la mise à jour de capacités de raisonnement disponibles plus précocement que ne le prévoyait le modèle piagetien. L'enfant disposerait des moyens logiques nécessaires à la comparaison verticale (entre la classe sur-ordonnée et la sous-classe), mais les propriétés de la situation le conduiraient à opérer une comparaison entre les sous-classes, via l'activation d'un schème de comparaison horizontale plus familier. La littérature expérimentale sur la quantification de l'inclusion peut être recensée à la lumière de cette analyse : la plupart des travaux ont en effet conduit à préciser la nature du facteur tâche en neutralisant les variables situationnelles potentiellement responsables de l'activation de ce schème « dangereux ». Les variables explorées sont globalement de trois ordres : perceptives, linguistiques et pragmatiques.

Rappelons que la situation classique met en jeu deux ensembles d'objets dont l'inégalité des extensions est perceptivement 
saillante. Plusieurs interprétations ont été proposées pour rendre compte de la manière dont ce contraste entre les deux sousclasses oriente l'attention de l'enfant vers une comparaison horizontale. Pour la plupart des auteurs, elle induit une attente liée à des routines cognitives de dénombrement et de comparaison d'ensembles d'objets indépendants. Pour Wilkinson (1976) la résolution de l'épreuve implique une procédure de double comptage (les objets A doivent être comptés une première fois en tant qu'ils appartiennent à la sous-classe des $A$, puis une seconde en tant qu'ils appartiennent également à la classe des B). Or cette procédure qui consiste à compter deux fois le même objet est proscrite dans l'apprentissage du dénombrement chez le jeune enfant. Il faut donc attendre que cette prohibition du double comptage se relâche pour que les sujets puissent appliquer la procédure les menant à une réponse correcte. Youssef et Guardo (1972) formulent pour leur part une hypothèse étonnante : c'est paradoxalement parce que la question d'inclusion leur apparaîtrait trop évidente que les enfants lui substitueraient une question de comparaison entre deux ensembles exclusifs. Quoi qu'il en soit, ces hypothèses envisagent toutes l'activation du schème de comparaison horizontale comme le produit d'une interaction entre le contraste perceptif d'une part et les routines cognitives du jeune enfant d'autre part. Afin de montrer que dans un contexte plus optimal les sujets seraient capables de mettre en cuvre un raisonnement correct, la manipulation des propriétés perceptives de la situation s'est principalement orientée dans deux directions. Une première direction a visé à réduire ou supprimer le contraste entre les sous-classes. C'est le cas des expériences faisant varier l'ampleur de la différence numérique entre les ensembles $A$ et $A^{\prime}$ (Ahr et Youniss, 1970 ; Kalil, Youssef et Lerner, 1974; Majeres et O'Toole, 1980), celles qui suppriment totalement le support perceptif en posant les termes du problème de manière exclusivement verbale (Wohlwill, 1968 ; Kurtz, 1978) ou de celles présentant les éléments des sous-classes mélangés plutôt que spatialement séparés (Wohlwill, 1968 ; Gold, 1987). La seconde direction de recherche a consisté à renforcer la prégnance perceptive de la classe sur-ordonnée pour faciliter la prise en compte de la relation d'inclusion. À la suite de Tatarsky (1974), plusieurs procédures ont ainsi été utilisées pour marquer visuellement l'appartenance des objets à une classe emboîtante en dotant tous les $A$ et les $A^{\prime} d^{\prime}$ 'une propriété 
perceptive commune (Kalil et al., 1974; Wilkinson, 1976 ; McCarrigle, Grieve et Hugues, 1978 ; Brainerd et Reyna, 1990 ; Chapman et McBride, 1992).

Un autre registre de travaux a permis de montrer que des modifications linguistiques dans la formulation de la question pouvaient affecter la propension des sujets à s'engager dans une comparaison verticale ou horizontale. La position de la classe sur-ordonnée dans la question a été manipulée (Kalil et al., 1974; Siegel, McCabe, Brand et Matthews, 1978), révélant un effet de récence sous certaines conditions. D'autres recherches se sont intéressées à la familiarité de l'enfant avec la catégorie surordonnée impliquée dans la relation d'inclusion (Kohnstamm, 1963 ; Siegel et al., 1978 ; Majeres et O'Toole, 1980), prolongeant ainsi les premières observations de décalage horizontal réalisées par Piaget et Inhelder (1959-1967) concernant la classe des fleurs et celle des animaux. Pour Carson et Abrahamson (1976), ce n'est pas tant le choix de la catégorie qui importe mais le choix des exemplaires composant les sous-classes. Ces exemplaires se différencient en effet selon leur niveau de typicité, c'est-à-dire leur force d'association à une catégorie donnée en vertu de leur proximité avec son prototype. Les auteurs ont montré que l'utilisation d'exemplaires atypiques favorisait une comparaison horizontale en permettant aux sujets de n'attribuer la propriété de $B$ qu'à l'une des deux sous-classes. À l'inverse, lorsque les sous-classes mentionnées sont toutes les deux typiques de la classe surordonnée, l'emboîtement est plus fréquemment pris en compte. Ce constat a par ailleurs conduit Lane et Hodkin (1985) à transformer cet effet en paradigme d'étude des représentations sociales. Il permet d'évaluer indirectement le niveau d'inclusion d'un concept dans une catégorie sociale. Les auteurs ont ainsi surpris les enfants américains en flagrant délit de stéréotypes sexuels, ces derniers transposant plus aisément la question : « Y a-t-il plus de femmes ou plus de docteurs? » en comparaison horizontale que la question : «Y a-t-il plus d'hommes ou plus de docteurs?" Markman et Seibert (1976) ont, pour leur part, montré que l'épreuve était mieux réussie lorsque la catégorie sur-ordonnée correspondait à une collection plutôt qu'à une classe. La collection, dont les éléments sont rassemblés sur la base d'une appartenance partitive au tout, présenterait une plus grande cohérence psychologique pour les jeunes enfants, facilitant ainsi la prise en compte de la relation d'emboîtement. Enfin, Shipley (1979) a 
montré que l'ajout de marqueurs linguistiques explicitant la comparaison requise conduit à augmenter la proportion de sujets traitant correctement la question, qui prend alors la forme suivante : « Which is more : All the animals or only the lions ? "

Un troisième registre de travaux a mis en avant les propriétés pragmatiques de la situation. Nous regroupons sous cette catégorie les manipulations expérimentales portant sur des facteurs susceptibles d'affecter l'interprétation par l'enfant de ce que l'expérimentateur attend de lui. Pour Politzer (1993, p. 264), l'épreuve de quantification de l'inclusion est en effet « particulièrement exigeante dans la mise à contribution des habiletés pragmatiques du sujet ». Cette dimension pragmatique trouve son origine dans l'asymétrie sociale de la relation, qui conduit l'enfant à interpréter les questions de l'adulte comme un test de compétence. Or, la quantification de classes exclusives (comme comparer $A$ et $A^{\prime}$ ) fait partie de ce qu'on attend fréquemment de lui en présence d'ensembles d'objets numériquement inégaux. Plusieurs procédures expérimentales ont ainsi cherché à rendre plus explicite pour le sujet l'attente de l'expérimentateur. Certaines études ont procédé à des comptages préalables (Wohlwill, 1968 ; Trabasso, Isen, Dolecki, McLanahan, Riley et Tucker, 1978 ; Judd et Mervis, 1979) ou à des demandes de comparaison horizontale (Youssef et Guardo, 1972 ; Winer, 1978 ; Carpendale, McBride et Chapman, 1996) avant de poser la question d'inclusion, afin de devancer une éventuelle interprétation erronée de la question critique. D'autres ont procédé à des mises en alerte comme Schwebel et Schwebel (1974) demandant aux enfants d'attendre quinze secondes avant de répondre, ou Winer et Faulkner (1984) en indiquant explicitement au sujet la nature piégeante de la question. Enfin, Ahr et Youniss (1970) puis Politzer (1993) ont également cherché à réduire les risques d'interprétation horizontale en spécifiant les trois termes dans la question ( « Y a-t-il plus de $A$ ou plus de $A^{\prime}$ ou plus de $B$ ? »).

\section{DES RÉSULTATS CONTRADICTOIRES}

Au total, ces diverses manipulations expérimentales ont produit un corpus de données extrêmement riche, mais contradictoire. Nous avons recensé ici les différentes variables envisagées 
sans nous intéresser précisément aux résultats obtenus. Plusieurs revues de question s'y sont efforcées et ont entrepris une comparaison systématique des résultats, notamment pour les revues les plus importantes, Winer (1980), Cormier et Laurendeau-Bendavid (1982), et Bideaud (1988). Il a ainsi été constaté à plusieurs reprises que des études manipulant le même facteur ne parvenaient pas à des conclusions identiques. Face à la confusion apparente des données, ces revues aboutissent au même constat : tous les facteurs proposés ont un effet sur les réponses des sujets mais aucun ne semble avoir d'effet systématique. Si la conclusion est identique au terme de ces trois revues, l'explication du phénomène varie.

Pour Winer (1980), l'absence d'homogénéité des résultats et l'impossibilité même de se prononcer sur un âge moyen de réussite à l'épreuve pourraient indiquer que des processus différents sont impliqués, à chaque âge, dans le traitement de la tâche. Il conviendrait également selon lui de s'intéresser à d'autres variables comme les styles cognitifs (principalement l'impulsivité et la dépendance à l'égard du champ), responsables de différences interindividuelles et susceptibles d'affecter les résultats. Pour Bideaud (1988), l'impossibilité d'isoler clairement les effets est liée à l'intrication des facteurs dans les plans expérimentaux. Il est en effet assez rare que deux études parviennent à maintenir à l'identique les dimensions à la fois perceptives, sémantiques et pragmatiques du dispositif de passation de l'épreuve. Cormier et Laurendeau-Bendavid (1982) vont pour leur part mettre l'accent sur une divergence majeure liée à l'utilisation de critères de réussite différents : alors que certains auteurs exigent une justification argumentée pour se prononcer sur le statut logique du raisonnement de l'enfant, d'autres se contentent d'une simple réponse correcte. On sait l'attachement de l'école genevoise à la prise en considération des arguments de l'enfant dans le diagnostic opératoire (Pascual-Leone et Bovet, 1966). La majorité des travaux postpiagetiens ont cependant suivi les recommandations méthodologiques de Brainerd (1973, 1977) pour qui l'exigence d'explicitation verbale pouvait amener à sous-estimer la compétence en question. L'adhésion massive (bien que souvent implicite) des auteurs à cette proposition a vraisemblablement conduit à l'inverse à introduire dans le corpus de données des false positives, liées à des réponses au hasard, notamment chez les plus jeunes enfants. Hodkin (1987) a montré que la litté- 
rature sur les compétences précoces en matière de raisonnement inclusif a, curieusement, négligé le fait qu'un choix aléatoire parmi les deux termes de la question conduirait à $50 \%$ de réussite. Elle propose alors un modèle d'analyse de la performance permettant de repérer ces réponses aléatoires. Ce modèle probabiliste a été récemment repris et sophistiqué par Thomas et Horton (1997). Nous considérons toutefois, avec Cormier et Laurendeau-Bendavid (1982), que ces détours expérimentaux pourraient être aisément évités par la simple prise en compte des justifications. Deux arguments viennent appuyer cette position. Chapman et McBride (1992) ont testé l'effet du mode d'évaluation sur la trajectoire développementale mise à jour. Ils ont ainsi contrasté les résultats obtenus lors d'une même expérience selon trois modes d'évaluation : la méthode Hodkin, le jugement simple et le jugement accompagné de justifications. Leurs résultats révèlent que la méthode Hodkin et l'analyse des justifications conduisent aux mêmes conclusions. D'autre part, Thomas et Horton (1997) eux-mêmes reconnaissent l'insuffisance d'un modèle probabiliste comme le leur pour identifier les variables développementales sous-jacentes à chacune des grandes stratégies identifiées. À l'inverse, l'analyse approfondie des justifications ne se contente pas uniquement de révéler l'existence de réponses aléatoires mais offre des pistes de compréhension (voir sur ce point Bideaud et Lautrey, 1983).

Si nous partageons les interprétations auxquelles ces trois revues de question ont abouti, nous pensons cependant qu'elles ont négligé un aspect susceptible d'éclairer également les apparentes contradictions de cette littérature : la prise en compte de l'interaction entre variables situationnelles et variables développementales. Il nous apparaît que la majorité des travaux mentionnés jusqu'alors sont de nature a-développementale. Au plan théorique, rappelons que malgré leur diversité de surface, ces manipulations expérimentales sont toutes orientées par une même hypothèse : confronté à l'épreuve de quantification de l'inclusion, le sujet ne parviendrait pas à faire la démonstration d'une compétence logique, dont pourtant il dispose, en raison de facteurs situationnels. Ces travaux ont donc montré que l'épreuve, dans son format traditionnel, est une situation piégeante, puisque plusieurs de ses propriétés (perceptives, linguistiques et pragmatiques) convergent vers l'activation d'un schème inadéquat de traitement. Mais ce faisant, ils ne 
s'interrogent aucunement sur les changements qui affectent le raisonnement de l'enfant de sorte que, à un point donné de son développement, la quantification de l'inclusion lui apparaît comme non ambiguë et sa solution logique évidente. Cet évitement de la question développementale s'exprime également dans le traitement des résultats. Des auteurs se sont opposés sur l'existence de certains effets situationnels en comparant des données recueillies sur des échantillons de sujets d'âges différents (voir par exemple les résultats contradictoires entre les études de Youssef et Guardo (1972) et Carpendale et al. (1996), concernant l'effet de questions préliminaires). Nous notons également que la plupart des expériences conduites sur des groupes d'âges contrastés donnent pourtant lieu à un traitement exclusif des données en termes d'effet simple de la variable situationnelle manipulée, sans prise en compte d'une éventuelle interaction avec l'âge. Il semble donc s'être opéré un consensus implicite pour penser que le «facteur tâche » devait, pour être reconnu, peser de manière équivalente dans la détermination de la performance à tous les niveaux de développement. Or, les rares études ayant considéré avec attention l'interaction entre variables expérimentales et développementales (Marantz, 1976 ; Kurtz, 1978 ; Brainerd et Reyna, 1990 ; Carpendale et al., 1996) semblent indiquer que ce n'est pas le cas et que, au contraire, la sensibilité du raisonnement de l'enfant aux variations contextuelles introduites est caractéristique de périodes critiques. La considération de cette interaction nous semble nécessaire pour expliquer la fluctuation des résultats en fonction des travaux ainsi que le caractère toujours partiel des effets mis à jour.

\section{LE STATUT COGNITIF \\ DE LA VARIABILITÉ INTRA-INDIVIDUELLE}

Une question essentielle soulevée par ce champ de recherche est celle du statut cognitif accordé à la variabilité des performances en fonction du contexte, de sa signification quant au développement logique sous-jacent. Pour Campbell et Bickhard (1986), l'introduction de la distinction compétence-performance a conduit les chercheurs à négliger l'étude des mécanismes de transformation de la connaissance en postulant l'existence d'une 
compétence formalisée a priori, et considérée comme définitivement disponible dès lors qu'un contexte d'évaluation donné permet de générer une proportion convaincante de réussite. Les travaux que nous venons d'évoquer illustrent manifestement cette orientation : la variabilité intra-individuelle de la performance est conçue comme résultant de biais méthodologiques, certains formats d'épreuves échouent à appréhender la compétence en question quand d'autres, à l'inverse, parviennent à la révéler. Les déterminants de cette variabilité intertâche sont alors situés à l'extérieur du sujet, laissant intacte la compétence supposée. Pourtant cette variabilité est elle-même l'objet d'une évolution développementale. Schröder et Edelstein (1991) ont conduit une étude longitudinale confrontant à 7,9 et 12 ans un même groupe de sujets à différentes épreuves d'inclusion. Plusieurs conditions étaient utilisées (matériel disponible ou problème posé verbalement) ainsi que diverses catégories sémantiques. Comme dans la plupart des études un « effet tâche » apparaît tel que certaines conditions génèrent en moyenne plus de réussite que d'autres. L'originalité de ce travail longitudinal est cependant de révéler une réduction de la variabilité intra-individuelle avec l'âge. Autrement dit, les réponses des enfants sont de plus en plus stables et de moins en moins sensibles aux variations contextuelles. Ce phénomène indique que la variabilité intertâche de la performance ne peut être réduite au statut de "bruit » expérimental mais qu'elle constitue en soi un fait dont les modèles développementaux devraient rendre compte. Nous rejoignons ici la position de Sophian (1997) pour qui la signification cognitive accordée à la variabilité constitue un enjeu transversal des recherches postpiagetiennes. Pour Sophian comme pour Campbell et Bickhard, le recours théorique à la distinction compétence-performance a donc orienté l'attention des chercheurs vers la construction de tâches optimales en réduisant considérablement leur préoccupation première : qu'est ce qui se développe ? Cette orientation résulte d'une focalisation exclusive sur le « facteur tâche » du modèle de Flavell et Wohlwill en négligeant les deux autres paramètres qui, eux, sont de nature développementale :

- Quelle forme prend la compétence sous-jacente à la résolution de l'épreuve?

- Comment rendre compte de la diminution de la sensibilité du sujet au facteur tâche? 
Si Flavell et Wohlwill ont dès le départ perçu la nécessité de fonder leur modèle sur l'interaction entre ces trois paramètres pour rendre compte des faits, la description qu'ils en proposent demeure cependant très vague. Si la compétence évolue entre ses formes premières de manifestation et sa forme aboutie, quelle est la nature de cette évolution? Si la sensibilité du sujet au facteur tâche évolue avec l'âge, à quel processus développemental en attribuer la responsabilité ? Deux registres d'hypothèses théoriques pour rendre compte de l'évolution des performances aux épreuves d'inclusion semblent se différencier précisément par leur centration sur l'un ou l'autre de ces paramètres et pourraient, ce faisant, nous aider à en préciser la nature.

\section{L'HYPOTHÈSE DU DÉFAUT DE COMPÉTENCE : DE LA RÉSOLUTION EMPIRIQUE À LA RÉSOLUTION LOGIQUE}

Alors que la grande majorité des recherches visaient à démontrer la disponibilité précoce de la compétence étudiée par Piaget, une série de travaux ont en revanche conduit à remettre en question le caractère logique des réponses obtenues dans l'épreuve de quantification de l'inclusion. Ce courant trouve notamment son origine dans l'utilisation d'une question additionnelle adressée à l'enfant ayant répondu correctement à la question classique. Cette nouvelle épreuve, proposée par Voelin (1976) et Markman (1978), consiste à demander au sujet si l'on peut faire quelque chose pour avoir plus de $B$ que de $A$ (par exemple, avec un matériel constitué de marguerites et de roses, « peut-on faire quelque chose pour avoir plus de marguerites que de fleurs? »). Autrement dit, l'enfant qui admet la supériorité numérique des $B$ sur les $A$ conçoit-il que cette supériorité ne peut être modifiée ? Un ensemble de résultats concordant révèle que cette épreuve dite « Modification » n'est guère réussie avant l'âge de 10-11 ans, soit bien plus tardivement que l'épreuve classique.

Pour Markman, les sujets qui répondent correctement à la question de quantification mais qui échouent à l'épreuve Modification (en répondant par exemple que l'on peut ajouter des 
marguerites) envisagent la supériorité de la classe sur-ordonnée comme un fait empirique qui doit être vérifié par les données car modifiable par leur transformation. Pour Voelin, ces sujets procèdent à une comparaison entre les étendues d'enveloppes spatiales correspondant aux ensembles à comparer. L'enveloppe des $B$ (constituée des $A$ et des $A^{\prime}$ réunis) ayant une étendue spatiale plus importante que l'enveloppe des A seuls, ces sujets concluent à la supériorité des $B$, mais ils conçoivent dans le même temps qu'il est possible d'inverser ce rapport en augmentant l'enveloppe des $A$ par adjonction d'éléments supplémentaires. Les travaux de Bideaud (1983; Bideaud et Lautrey, 1988) ont renforcé la distinction entre deux modes de résolution, l'un empirique (correspondant à la comparaison de collections disjointes) et l'autre logique. Une analyse détaillée des justifications utilisées par les enfants pour étayer leurs réponses à la question classique a révélé plusieurs catégories d'arguments. Certains semblent indiquer un traitement empirique de la situation fondé sur la comparaison d'enveloppes spatiales (« les fruits c'est tout ça et les pommes que ça » accompagné de gestes entourant les collections) ou la comparaison numérique associée à une procédure de dénombrement ( « il y a 5 fleurs au total et seulement 2 marguerites »). D'autres, comme l'argument d'appartenance ( « il y a plus de fleurs parce que les marguerites et les roses sont des fleurs »), semblent en revanche plus proche d'un traitement logique. Bideaud et Lautrey (1983) ont constaté que l'argument d'appartenance est celui qui apparaît le plus tardivement dans les justifications des enfants de différents niveaux d'âge et que les sujets qui utilisent cet argument sont plus nombreux que les autres à réussir l'épreuve Modification.

Pour tous ces auteurs (Voelin, Markman ou Bideaud), l'épreuve de quantification de l'inclusion ne peut donc plus être considérée comme l'épreuve critique de la compétence logique étudiée puisqu'elle peut être résolue par des moyens non logiques (traitement de collections disjointes et non de classes en relation d'emboîtement). Reste toutefois à expliquer les mécanismes développementaux qui assurent la transition entre les nouvelles étapes ainsi révélées : sujet non incluant (échec à l'épreuve classique), sujet incluant empirique (réussite à l'épreuve classique mais échec à Modification) et sujet incluant logique (réussite aux deux épreuves). Pour Bideaud et Houdé 
(1989), cette transition observée dans les conduites aux épreuves d'inclusion pourrait être l'expression d'une transformation sousjacente des représentations catégorielles et, plus précisément, d'un passage de représentations de type schématique (où les éléments sont rassemblés entre eux sur la base de relations de contiguïté) à des représentations de type taxonomique (organisées selon des relations de substituabilité). Cette interprétation a par la suite été nuancée par la découverte d'une disponibilité plus précoce qu'on ne le supposait du mode de catégorisation taxonomique, Houdé mettant alors l'accent sur les processus attentionnels permettant de réguler la mobilisation de modes concurrents (voir plus loin).

Le cadre général d'analyse partagé par ces différents travaux est celui d'un défaut de compétence logique et conduit à en repousser tardivement l'âge d'apparition. Mais jusqu'où ? Pour Barrouillet (1993) l'approche adoptée peut conduire à différer indéfiniment le critère de logicité à la « dernière tâche en date » et ce, jusqu'au raisonnement adulte. Ses propres travaux ont en effet révélé que la réussite à l'épreuve Modification ne constituait pas nécessairement le terme du développement de la logique des classes: de nouvelles tâches demandant au sujet d'aménager lui-même des configurations d'objets illustrant des classes en relation d'emboîtement sont réussies plus tardivement. Le choix de l'épreuve critique de la compétence relèvera toujours de l'arbitraire ou des préconceptions du chercheur, dès lors que l'approche du développement conceptuel retenue demeure formulée en termes de tout ou rien. La limite théorique de cette approche, dénoncée à plusieurs reprises (Campbell et Bickhard, 1986 ; Blewitt, 1989 ; Montangero, 1991 ; Barrouillet, 1993 ; Sophian, 1997) tient à sa conception binaire de la compétence : elle est disponible ou ne l'est pas.

Plus récemment, un nouveau cadre d'analyse des discordances compétence-performance a été proposé. Il se fonde sur le développement des capacités de résistance à l'interférence chez l'enfant et, ce faisant, tend à rendre compte du troisième paramètre du modèle de Flavell et Wohlwill : la sensibilité du sujet au facteur tâche. 
L'HYPOTHĖSE D'UNE RÉDUCTION

DE LA SENSIBILITÉ AU FACTEUR TÂCHE : LE DÉVELOPPEMENT DE L'INHIBITION COGNITIVE

Au terme de sa revue de littérature, Winer (1980) suggérait que l'évolution des performances à l'épreuve d'inclusion puisse être liée à des processus cognitifs indépendants de la logique des classes, mais dont le développement parallèle affecterait le traitement de l'épreuve. C'est la perspective adoptée par la plupart des modèles néo-piagétiens (Case, 1985 ; Halford, 1992 ; et PascualLeone, 1988, pour les plus cités) qui subordonnent l'élaboration des structures de connaissance logiques au développement des capacités attentionnelles de la mémoire de travail. Bien que ces modèles diffèrent dans leur conceptualisation des ressources attentionnelles et dans les métriques adoptées pour leur évaluation, tous partagent l'hypothèse d'un développement conceptuel contraint par le caractère limité de ces ressources. Barrouillet (1993) a montré que les propositions de Case (1985) pouvaient être adaptées au domaine de la quantification de l'inclusion, permettant de rendre compte des étapes qualitatives de son développement par des changements d'ordre quantitatif, relatifs à l'évolution des capacités de traitement. Ainsi, les progrès observés chez l'enfant dans la compréhension des systèmes de liaison entre la classe emboîtante et les sous-classes pourraient résulter d'une coordination croissante de dimensions jusqu'alors appréhendées de manière isolée. Outre l'accent mis sur ces contraintes en termes de capacités d'activation et de coordination des structures de connaissance, le modèle de Pascual-Leone intègre également un opérateur de désactivation (i.e. d'inhibition) dont le rôle fait aujourd'hui l'objet d'un éclairage nouveau. Depuis le début des années 1990, plusieurs propositions théoriques ont, en effet, mis l'accent sur le rôle du processus d'inhibition cognitive dans la résistance aux phénomènes d'interférence en mémoire de travail (Hasher et Zacks, 1988 ; Bjorklund et Harnishfeger, 1990 ; Dempster et Brainerd, 1995, pour les contributions les plus importantes). Le processus d'inhibition y est conçu comme un mécanisme de suppression active permettant la sélection des représentations appropriées à la tâche en cours parmi l'ensemble de celle préactivées par le contexte. 
Pour Houdé (1995), la plupart des grands paradigmes de la psychologie développementale constituent des situations pièges dont la résolution n'impliquerait pas seulement des capacités d'activation ou de coordination des schèmes pertinents, mais également des capacités de résistance à l'interférence, via l'inhibition des schèmes non pertinents. Ce cadre d'analyse, fondé sur la prise en compte des contraintes d'inhibition inhérentes aux épreuves développementales, place la résistance du sujet à l'interférence au cour de l'explication des discordances compétence-performance.

Les discordances émergeraient à des étapes développementales où, alors que l'enfant dispose de la structure rationnelle évaluée par l'épreuve, le processus d'inhibition encore insuffisamment efficient ne permet pas de résister aux schèmes dangereux activés par les propriétés du contexte. Des situations dites optimales, c'est-à-dire épurées des facteurs situationnels responsables de l'activation de ces schèmes, permettraient en revanche l'expression de compétences logiques précocement disponibles. L'évolution « turbulente » de la performance des enfants aux épreuves d'inclusion peut selon Houdé être expliquée par les contraintes d'inhibition inhérentes à chacune des épreuves utilisées. L'épreuve Modification requiert en effet l'inhibition d'une routine arithmétique familière selon laquelle, pour avoir plus d'éléments dans un ensemble, il suffit d'en ajouter. Les sujets inhibiteurs inefficients céderaient alors à ce schème dangereux en considérant que, pour avoir plus de marguerites que de fleurs, on peut rajouter des marguerites. Dans cette perspective, l'échec à l'épreuve modifiée d'inclusion ne signalerait pas un défaut de compétence logique (comme c'est le cas des interprétations évoquées dans le point précédent) mais bien plus un défaut de programmation exécutive de l'inhibition. Une analyse équivalente peut être appliquée à la résolution de l'épreuve classique. L'épreuve de quantification de l'inclusion a en effet été décrite comme une situation piège, en ce sens que plusieurs de ses propriétés convergent vers l'activation d'un schème de comparaison horizontale, susceptible de court-circuiter la mise en œuvre d'un raisonnement inclusif approprié. Les divers aménagements expérimentaux recensés seraient alors à considérer comme des situations optimales qui, en réduisant la prégnance des propriétés activatrices de ce schème et/ou en renforçant l'activation d'une comparaison verticale, permettraient à l'enfant encore 
inhibiteur inefficient de faire la démonstration de sa compétence logique. Une telle conception met l'accent, non pas sur une transformation de la compétence sous-jacente, mais sur le développement parallèle de processus de contrôle exécutif qui assurent une insensibilité croissante au facteur tâche.

Ce nouveau cadre d'analyse nécessite toutefois d'être précisé sur un aspect important. Le modèle d'Houdé (1995) envisage l'émergence précoce d'une compétence logique dans le domaine du raisonnement inclusif, cette compétence demeurant inchangée entre ses premières formes de manifestation dans les épreuves optimales et ses formes ultimes d'expression dans les épreuves plus exigeantes et réussies plus tardivement. La réduction de la variabilité intra-individuelle de la performance en fonction des épreuves utilisées est attribuée au processus d'inhibition dont le développement est conçu comme indépendant de celui des structures de connaissance sur lesquelles il opère. Qu'est-ce qui, dès lors, assure l'efficience croissante du processus?

Pour les modèles anglo-saxons (Bjorklund et Harnishfeger, 1990 ; Dempster, 1991), le développement de l'inhibition est clairement conçu comme résultant de facteurs maturatifs d'origine cérébrale. C'est donc une capacité basique d'inhibition qui se développe et l'on doit pouvoir en observer les effets de manière synchronique sur une large variété d'épreuves d'interférence. Dans le modèle d'Houdé en revanche, ce qui se développe est la programmation exécutive de l'inhibition sans que le modèle ne précise explicitement l'origine de ce développement. Nous pouvons toutefois inférer des dispositifs expérimentaux utilisés par l'auteur qu'il ne saurait se réduire à une dimension maturative. Un des recours méthodologiques privilégié par Houdé dans la mise à jour des contraintes d'inhibition consiste en effet à réaliser un « apprentissage expérimental à l'inhibition ». Il s'agit de contraster la performance des sujets à une tâche de raisonnement avant et après avoir attiré leur attention sur les aspects piégeant de la tâche utilisée. Si un dispositif d'apprentissage permet de lever le défaut de programmation exécutive initialement observé, c'est que la probabilité d'une telle programmation ne dépend pas uniquement de facteurs maturatifs.

Pour Camus (1996, p. 134-135), la programmation de l'inhibition est ainsi étroitement dépendante de la reconnais- 
sance du caractère localement dangereux des schèmes : «Il semble légitime d'inhiber une conduite à partir du moment où son inadaptation est reconnue [...] Du point de vue développemental, la question qui est alors posée est de savoir si l'enfant dispose de ces processus d'identification du caractère inadapté de certaines de ces conduites. "De manière plus générale, peuton concevoir que l'inhibition se développe sans qu'aucun changement n'affecte la nature de la compétence logique sous-jacente (comme le laisse supposer la formalisation adoptée par Houdé) ? Selon Charron (1998), il est nécessaire d'articuler les deux cadres interprétatifs (transformation des connaissances et adaptation des stratégies attentionnelles d'activation/inhibition). L'articulation proposée envisage le dépassement d'une discordance compétence-performance comme résultant d'une « transformation de la connaissance qui consiste à modifier le domaine de validité de savoirs antérieurs en même temps que s'adaptent les fonctions exécutives " $(1998$, p. 7$)$. Autrement dit, l'enfant (tout comme l'adulte dans les dispositifs d'apprentissage expérimental à l'inhibition) dispose d'un répertoire de schèmes et l'évolution de ses performances est fonction de sa capacité à estimer la pertinence de l'application d'un schème en fonction des exigences du contexte. Cette perspective renvoie clairement le dépassement des discordances à un niveau métacognitif. C'est d'ailleurs la position adoptée plus explicitement par Houdé $(2000$, p. 141) dans ses formulations les plus récentes ( « les changements exécutifs sont métacognitifs, et non pas cognitifs, dans le sens où le contrôle inhibiteur et le set-shifting dépendent d'une métareprésentation de l'acte habituel comme inadapté »).

Une telle conception laisse toutefois entière la question du processus développemental qui conduit à cet ajustement normatif du domaine de validité des schèmes : comment et pourquoi le sujet en vient-il à considérer, à un moment de son développement, que les schèmes dangereux qu'il appliquait jusqu'alors sont inadaptés au traitement de la situation ? Cette question ne peut selon nous être résolue si l'on maintient la conception (inhérente au modèle d'Houdé et préservée dans les propositions de Charron) selon laquelle la connaissance logique demeurerait inchangée à chacune des étapes du développement du raisonnement inclusif. 


\section{DÉVELOPPEMENT LOGIQUE ET NIVEAUX DE CONNAISSANCES}

L'ensemble des cadres explicatifs que nous avons présentés ont en commun qu'ils préservent une approche dichotomique du développement logique (caractérisé en termes de présence/absence de la compétence). Cette représentation dichotomique, héritée du modèle piagetien, a continué de prévaloir malgré l'abandon d'un structuralisme strict, les auteurs s'opposant certes sur le moment où situer la rupture épistémologique mais non sur son existence. Or, nous pensons qu'une telle conception est à l'origine des principaux écueils théoriques rencontrés par ces modèles et que, par conséquent, une approche en termes de « niveaux de connaissances» serait plus à même de rendre compte des étapes qualitatives du développement logique. Nous partageons sur ce point l'analyse de Montangero (1991) pour qui la reconnaissance d'une hiérarchie de niveaux de connaissance constitue le moyen privilégié pour sortir enfin de la controverse sur les critères de compétence. Plusieurs modèles généraux du développement cognitif intègrent cette dimension: Moshman (1982; Moshman et Timmons, 1990), Campbell et Bickhard (1986), Karmiloff-Smith (1992, 1994). C'est le cas également des derniers travaux de Piaget sur les morphismes dont Barrouillet (1993; Barrouillet et Poirier, 1997) a montré qu'ils étaient compatibles avec une telle approche.

Ces différents modèles partagent plusieurs propriétés. La principale est que le développement ne procède pas d'une rupture entre un sujet irrationnel et un sujet rationnel mais d'une succession de niveaux de connaissances qui entretiennent entre eux des rapports de filiation. L'objectif théorique est alors d'une part de décrire précisément chaque niveau de connaissance et le type de performance qu'il autorise, mais surtout de comprendre le processus développemental assurant la transition entre ces niveaux. L'enfant expérimente dans ses interactions avec le monde physique et social des systèmes de relations qui relient entre eux des états. Ces liaisons peuvent être données par la perception (c'est le cas des morphismes piagetiens) ou par le repérage d'une régularité entre l'action engagée par le sujet et le but qu'elle permet d'atteindre (maîtrise procédurale pour Karmiloff- 
Smith, interaction efficace pour Campbell et Bickhard). Quel que soit le domaine conceptuel étudié, le premier niveau de connaissance procède donc toujours d'une abstraction des régularités observées dans l'environnement. Pour qu'un développement s'opère, c'est-à-dire une transformation qualitative de la connaissance et non une simple accumulation de schèmes de même niveau, il est nécessaire qu'un second niveau de connaissance prenne le premier pour objet. L'abstraction réfléchissante (Piaget, 1977 ; Campbell et Bickhard, 1986) tout comme la redescription représentationnelle (Karmiloff-Smith, 1992) correspondent à un processus par lequel les propriétés implicites du premier niveau deviennent l'objet de connaissance explicite du niveau supérieur. Le processus implique un déplacement de l'attention du sujet, du traitement des propriétés de l'environnement vers le traitement des propriétés de ses propres procédures.

Ce processus récursif permet selon Moshman (1990) de rendre compte de deux caractéristiques essentielles du raisonnement logique : la généralisation (le raisonnement n'est plus dépendant des contenus empiriques auxquels il s'applique) et la nécessité. En effet, la nécessité logique, qui caractérise les niveaux supérieurs de connaissance, n'est pas une propriété de l'environnement donnée dans les faits mais une propriété implicite des transformations qui peuvent lui être appliquées ( $X$ est nécessaire, non parce qu'on constate que $X$, mais parce qu'aucune transformation ne peut faire que non- $X$ ). Il nous semble dès lors qu'un modèle du développement du raisonnement inclusif devrait pouvoir décrire comment le sujet parvient à construire, par abstractions successives, un système de relations générales et nécessaires entre les extensions des classes et des sous-classes.

\section{VERS UNE INTÉGRATION DES CADRES D'ANALYSE}

Chacune des conceptions théoriques évoquées jusqu'ici est supportée par des résultats expérimentaux convaincants. Il nous faut donc admettre que même si elles ont été formulées en des termes souvent exclusifs, elles appréhendent toutes une part de la réalité du développement du raisonnement logique. Ce faisant, elles fournissent des contraintes utiles à toute tentative de 
modélisation de ce développement. La distinction introduite entre les modes de résolution empirique et logique a constitué une avancée décisive dans la compréhension du raisonnement inclusif. Elle relève d'une conception plus générale selon laquelle l'identité de la performance n'équivaut pas nécessairement à une identité du niveau de conceptualisation sous-jacent (Sophian, 1997). Nous pensons également avec Houdé (1995) que le développement ne se réduit pas seulement à la « substitution majorante d'une structuration rationnelle à une structuration qui ne l'est pas ou qui l'est moins ". L'expression des capacités de raisonnement logique est subordonnée, dans les situations pièges, aux phénomènes de compétition entre structures de connaissances concurrentes dont l'inhibition constitue un processus régulateur. La proposition qui suit, structurée autour de trois principales étapes développementales, cherche à réintégrer ces approches. La conception avancée ici vise, en suivant les recommandations de Montangero (1991) et de Barrouillet (1993), à dépasser l'approche binaire de la compétence qui prévaut dans les modèles précédents. Cette conception repose sur la discrimination de niveaux de connaissance qui dérivent les uns des autres par le jeu d'abstractions réfléchissantes successives. Elle vise, dans le même temps, à rendre compte des progrès de l'inhibition dans les situations pièges par une reconnaissance croissante de l'inadaptation des schèmes dangereux antérieurement appliqués.

NIVEAU 1

Plusieurs travaux de Piaget (Piaget, 1977 ; Piaget et Garcia, 1987) ont montré que, avant de pouvoir résoudre le problème de quantification, l'enfant était capable de manipuler, sur le plan intensionnel, des relations d'inclusion entre catégories. Pour Piaget, cette capacité est fondée sur la maîtrise des implications signifiantes : il existe des liens suffisamment constants entre les propriétés qualitatives des objets pour permettre au sujet d'inférer la présence de l'une à partir de la perception d'une autre (i.e. si un objet possède l'attribut « marguerite ", alors il possède également l'attribut « fleur »). Cette implication signifiante, qui conduit à pouvoir inclure un même objet dans des catégories multiples à différents niveaux de généralité, est le 
produit d'une première abstraction sur les propriétés des objets. Plusieurs travaux expérimentaux (Greene, 1994; Houdé et Charron, 1995) ont confirmé que, dès 5-6 ans, les enfants étaient en effet capables d'utiliser des relations d'inclusion entre catégories ainsi que certaines propriétés de cette organisation hiérarchique comme la transitivité des significations. Pourtant ce premier niveau de connaissance n'entraîne pas la réussite à l'épreuve de quantification de l'inclusion. Sur le plan des performances, il correspond à une réussite aux épreuves de logique intensionnelle mais qui est associée à un échec à toutes les épreuves de quantification des extensions: les sujets assimilent la question de quantification de l'inclusion à une comparaison de l'extension des sous-classes. Pour Houdé et Charron (1995), ce sujet «logicien intensionnel » disposerait des moyens logiques nécessaires au traitement de l'épreuve mais échouerait à résister au piège extensionnel que constitue la supériorité perceptivement saillante des $A$ sur les $A^{\prime}$. Si cette analyse est pertinente, elle laisse toutefois en suspens, comme nous l'avons discuté précédemment, la question de l'origine de ce défaut d'inhibition, et donc de son dépassement ultérieur. Une solution possible revient à considérer que la maîtrise des implications signifiantes ne constitue pas le terme du développement logique mais un premier niveau de connaissance sur lequel vont opérer les abstractions réfléchissantes ultérieures. À ce niveau, le schème de comparaison horizontal n'est pas inhibé parce qu'il constitue encore le seul outil de traitement des extensions à la disposition du sujet et, par conséquent, son inadéquation avec l'exigence de la question d'inclusion ne peut être reconnue.

NIVEAL 2

La comparaison de l'extension de $A$ et de celle de $B$ implique le double comptage des objets qui composent $A$. La nécessité de ce double comptage est une propriété, au plan extensionnel, de l'appartenance simultanée des objets à la sous-classe et à la classe emboîtante, laquelle est maîtrisée au niveau précédent. L'accès à ce second niveau de connaissance est ainsi assuré par une abstraction réfléchissante sur les propriétés de l'implication signifiante du niveau 1 . Cette abstraction conduit le sujet à élaborer un schème de double comptage dont l'application lui per- 
met de parvenir à une réponse correcte à la question de quantification de l'inclusion. La notion de double comptage ne renvoie pas ici nécessairement à un dénombrement exact des collections. Le sujet peut opérer une estimation globale des extensions par une comparaison de leurs enveloppes spatiales, comme l'a décrit Voelin (1976). Ce niveau présente encore toutefois deux limites importantes. La résolution de l'épreuve relève de l'application attentionnelle d'un schème de double comptage (ou de construction de deux enveloppes) qui opère sur les données disponibles. $L$ 'activation de ce schème nouvellement construit et l'inhibition du schème concurrent et familier de comparaison horizontale sont alors étroitement dépendantes de l'organisation de ces données et, plus généralement, des propriétés situationnelles discutées précédemment. D'autre part, la supériorité de l'extension des $B$ relève d'un constat empirique issu de l'application du schème pertinent. Ce niveau de connaissance est donc caractérisé par la possibilité de modifier ce constat en modifiant les données. Il en résulte un échec systématique à l'épreuve Modification où le sujet applique le « schème d'ajout et de retrait » (pour avoir plus de $A$ que de $B$, il faut ajouter des $A$ ). Un nouveau pas d'abstraction sera nécessaire pour lui permettre de reconnaître l'inadéquation de ce schème car, à ce niveau, la conclusion à laquelle il aboutit $(B>A)$ ne présente aucunement un caractère nécessaire.

NIVEAU 3

La généralité et la nécessité de la supériorité de l'extension de $B$ sur celle de $A$ sont une propriété qui découle de la procédure de double comptage dont l'application est maîtrisée au niveau précédent : c'est parce que tout élément de A est également comptabilisé en tant que $B$ qu'une modification de l'extension de $A$ s'accompagne nécessairement d'une modification de l'extension de $B$. L'accès à ce troisième niveau de connaissance est issu d'une abstraction réfléchissante sur les propriétés du double comptage opéré au niveau 2. Cette abstraction correspond bien, comme le décrivent les modèles de Piaget, Campbell et Bickhard ou Karmiloff-Smith, à un déplacement de l'attention : alors qu'au niveau précédent l'attention du sujet est dirigée vers le traitement des propriétés des objets, elle est ici 
orientée vers le traitement des propriétés de ses propres procédures. Le sujet abstrait alors de ces propriétés un système de relations entre les extensions des classes et des sous-classes qui confère à la supériorité de $B$ sur $A$ un caractère général et nécessaire. Le traitement des différentes épreuves d'inclusion s'en trouve modifié. La résolution de l'épreuve classique de quantification ne relève plus de l'application d'un schème de double comptage aux données empiriques mais d'une inférence fondée sur la connaissance $d$ 'un système de relations. Ce système est de nature générale et assure une indépendance du raisonnement vis-à-vis des aspects de contenu, permettant au sujet de résoudre les problèmes formulés verbalement, et quel que soit le dispositif de passation de l'épreuve (réduction de la variabilité intertâche). Enfin, la nécessité logique de la supériorité de l'extension de $B$ sur celle de A (abstraite des propriétés du double comptage) permet la reconnaissance du caractère inadapté du «schème d'ajout et de retrait " dans le traitement de l'épreuve Modification, autorisant ainsi la programmation exécutive de son inhibition.

Bien que peu de travaux l'aient envisagé, un quatrième niveau pourrait être distingué, procédant d'une abstraction réfléchissante sur les propriétés du système de relations entre les extensions des classes et sous-classes (maîtrisé au niveau 3). Un pas supplémentaire d'abstraction est en effet nécessaire pour que le sujet conçoive que si l'extension de $B$ est nécessairement supérieure à celle de $A$, l'extension des non-A est nécessairement supérieure à celle des non-B (Piaget, 1977 ; Müller, Sokol et Overton, 1999).

D'un point de vue théorique, cette conception permet l'articulation des cadres interprétatifs précédents tout en dépassant certains obstacles. Le modèle d'Houdé a posé la question du rapport de causalité entre les facteurs explicatifs de la performance : « Les sujets sont-ils incapables d'inhiber parce qu'ils ne sont pas rationnels [...] ou apparaissent-ils irrationnels parce qu'ils sont incapables d'inhiber ? " (1999, p. 274, notre traduction). Ces deux propositions ne sont plus nécessairement exclusives dès lors qu'on distingue deux sources possibles au défaut d'inhibition :

métacognitive : l'inhibition n'est pas programmée parce que le niveau de développement logique ne permet pas encore de reconnaître le caractère localement inadapté du schème dan- 
gereux. Nous avons proposé que les progrès exécutifs (la résistance aux situations pièges) étaient contraints par des niveaux de connaissance distincts, autorisant ou non cette reconnaissance ;

- attentionnelle : à niveau de connaissance équivalent, l'efficience du processus de suppression d'informations varie en fonction des caractéristiques attentionnelles du sujet. Les recherches actuelles sur le processus (et non sur les déterminants logiques de sa programmation) montrent que son efficience est en effet sensible à de nombreux facteurs de variation : évolution développementale (Kipp Harnishfeger, 1995), différences interindividuelles (Kipp Harnishfeger et Bjorklund, 1994), styles cognitifs (Visser et al., 1996), psychopathologie (Enright et Beech, 1993), ainsi qu'à des variations intra-individuelles en fonction du niveau d'éveil attentionnel (Hasher, Zacks et May, 1999).

Notre proposition théorique vise à rendre compte des progrès observés dans le registre métacognitif de la programmation de l'inhibition. D'un point de vue empirique, cette conception devra conduire à reconsidérer avec attention les patterns de variabilité intra-individuelle de performance et leur évolution. Dans cette perspective en effet, la fluctuation des performances en fonction des modalités d'évaluation d'un même concept ne constitue plus un phénomène paradoxal ou discordant. À l'inverse, elle devient l'indicateur privilégié d'une hiérarchie de niveaux de connaissance qu'elle révèle.

\section{CONCLUSIONS}

Nous avons souligné l'importance de la prise en compte des discordances compétence-performance dans le développement logique chez l'enfant. Le recours à la distinction introduite par Flavell et Wohlwill (1969) a permis de révéler les contraintes exécutives qui pèsent sur la performance, mais elle a également conduit à garantir les modèles de compétence contre toute remise en cause théorique ou empirique de leur validité (Campbell et Bickhard, 1986; Sophian, 1997) et, par-là, à relayer au second plan le développement logique proprement dit. Faut-il, comme le propose Russel (2000), abandonner cette distinction et 
réintégrer le contrôle exécutif dans la notion même de compétence? Au terme de cette revue théorique, il apparaît que la problématique des compétences précoces et des incompétences tardives questionne avant tout notre représentation de la compétence. Elle repose fréquemment, comme nous l'avons vu, sur une conception statique formulée en termes de disponibilité ou non d'une connaissance définitive formalisée a priori par le chercheur. Il nous semble aujourd'hui nécessaire de reconnaître l'existence, au sein d'un même domaine conceptuel, d'une hiérarchie de niveaux de rationalité, caractérisés par des exigences distinctes en matière de contrôle exécutif.

\section{RÉSUMÉ}

Cet article explore, à travers l'exemple de la quantifícation de l'inclusion, les principaux cadres théoriques avancês pour rendre compte des discordances compétence-performance au cours du développement cognitif. Leur recension critique indique que les hypothèses se sont successivement focalisées sur chacun des trois paramètres spécifiés dans le modèle initial de Flavell et Wohlwill (1969). Un premier courant de recherche a clairement démontré l'existence d'une variabilité intra-individuelle des performances en fonction des propriétés des épreuves utilisées. Un second, ayant recours à des épreuves aux exigences logiques accrues, rend compte de cette variabilité par un défaut de compétence logique, laquelle n'émergerait pas avant l'adolescence. Pour un troisième courant, la compétence logique serait à l'inverse précocement disponible, les discordances résultant d'un défaut d'inhibition de schèmes inadaptés. Après une discussion des diffícultés théoriques rencontrées par ces hypothèses alternatives, nous suggérons qu'une approche en termes de " niveaux de connaissance " permettrait leur articulation.

Mots-clés : développement cognitif, compétence-performance, inhibition, niveaux de connaissance, quantification de l'inclusion.

\section{BIBLIOG RAPHIE}

Ahr P. R., Youniss J. - (1970) Reasons for failure on the class inclusion problem, Child Development, 41, 131-143.

Barrouillet P. - (1993) Du bricolage à la logique chez l'enfant? Line approche structuraliste de la genèse de l'inclusion, L'Année psychologique, 9.3, 283310.

Barrouillet P., Poirier I. - (1997) Comparing and transforming : An application of Piaget's morphisms theory to the development of class inclusion and arithmetic problem solving, Human Development, 40, $216-234$.

Bideaud J. - (1988) Logique et bricolage chez l'enfant, Lille, Presses Iiniversitaires de Lille. 
Bideaud J., Houdé O. - (1989) Le développement des catégorisations : « capture » logique ou « capture » écologique des propriétés des objets, $L$ 'Année psychologique, 89, 87-123.

Bideaud J., Lautrey $J$. - (1983) De la résolution empirique à la résolution logique du problème d'inclusion : évolution des réponses en fonction de l'âge et des situations expérimentales, Cahiers de psychologie cognitive, 3, 295-326.

Bjorklund D. F., Kipp Harnishfeger K. - (1990) The resources construct in cognitive development : Diverse sources of evidence and a theory of inefficient inhibition, Developmental Review, 10, 48-71.

Blewitt P. - (1989) Categorical hierarchies : Levels of knowledge and skill, The Genetic Epistemologist, 17, 21-29.

Brainerd C. J. - (1973) Judgments and explanations as criteria for the presence of cognitive structures, Psychological Bulletin, 79, 172-179.

Brainerd C. J. - (1977) Response criteria in concept development, Child Development, 48, 360-366.

Brainerd C. J., Reyna V. F. - (1990) Inclusion illusions : Fuzzy-trace theory and perceptual salience effects in cognitive development, Developmental Review, 10, 365-403.

Campbell R. L., Bickhard M. H. - (1986) Knowing levels and developmental stages, New York, Karger.

Camus J.-F. - (1996) La psychologie cognitive de l'attention, Paris, Armand Colin.

Carpendale J. I., McBride M. L., Chapman M. - (1996) Langage and operations in children's class inclusion reasoning: The operational semantic theory of reasoning, Developmental Review, 16, 391-415.

Carson M. T., Abrahamson A. - (1976) Some members are more equal than others : The effect of semantic typicality on class inclusion performance, Child Development, 47, 1186-1190.

Case R. - (1985) Intellectual development : Birth to adulthood, New York, Academic Press.

Chapman M., McBride M. L. - (1992) Beyond competence and performance : Children's class inclusion strategies, superordinate class cues, and verbal justifications, Developmental Psychology, 2, 319-327.

Charron C. - (1998) Ruptures et continuïté dans la construction des nombres, thèse de doctorat, Université René-Descartes, Paris.

Cormier P., Laurendeau-Bendavid M. - (1982) La considération des justifications : un moyen de sortir de l'impasse les recherches sur la quantification de l'inclusion, Cahiers de psychologie cognitive, 2, 373-388.

Dempster F. N. - (1991) Inhibitory processes : A neglected dimension of intelligence, Intelligence, 15, 157-173.

Dempster F. N., Brainerd C. J. - (1995) Interference and inhibition in cognition, New York, Academic Press.

Enright S. J., Beech A. - (1993) Reduced cognitive inhibition in obsessivecompulsive disorder, British Journal of Clinical Psychology, 32, 67-74.

Flavell J. H., Wohlwill J. F. - (1969) Formal and functional aspects of cognitive development, in D. E. Flavell (Edit.), Studies in cognitive development : Essays in honor of Jean Piaget, New York, Oxford Press.

Gold R. - (1987) Class inclusion performance : Effect of intermingling the subsets, British Journal of Developmental Psychology, 5, 343-346.

Greene T. R. - (1994) What kindergartners know about class inclusion hierarchies, Journal of Experimental Child Psychology, 57, 89-107. 
Halford 6. - (1992) Analogical reasoning and conceptual complexity in cognitive development. Human Development, 35, 193-217.

Hasher I.., Zacks R. T. - (1988) Working memory, comprehension, and aging : A review and a new view, in (;. H. Bower (Edit.), The psychology of learning and motivation, San Diego, Academic Press, 193-225.

Hasher L., Zar:ks R. T., May C. P. - (1999) Inhibitory control, circadian arousal, and age, in D. (jopher et A. Koriat (Edit.), Attention and performance. Cognitive regulation of performance : Interaction of theory and application, Cambridge, MIT Press, 653-675.

Hodkin B. - (1987) Performance model analysis in class inclusion : An illustration with two langage conditions, Developmental Psychology, 23, 683-689.

Houdé (). - (1995) Rationalité, développement et inhibition., Paris, Plif.

Houdé O. - (1999) Executive performance/competence, and inhibition in cognitive development. Object, number, categorization and reasoning, Developmental Science, 2, 273-275.

Houdé O. - (2000) Inhibition and cognitive development : Object, number, categorization and reasoning, Cognitive Development, 15, 63-73.

Houdé $0 .-(2000)$ I a genèse de la cognition. L'esprit piagétien et les perspectives actuelles, in 0 . Houdé et (C. Meljac (Edit.), L'esprit piagétien. Hommage international à Jean Piaget, Paris, PtF, 127-148.

Houdé 0 ., Charron $C$. - (1995) Catégorisation et logique intensionnelle chez l'enfant, L'Année psychologique, 95, 63-86.

Howe M. I., Rabinowitz M., Powell T. I. - (1998) Individual differences in working memory and reasoning-remembering relationships in solving classinclusion problems, Memory and Cognition, 26, $1089-1101$.

Judd S. A., Mervis C. B. - (1979) learning to solve class-inclusion problems : The roles of quantification and recognition of contradiction, Child Development, 50, 163-169.

Kalil K., Youssef Z., Lerner R. - (1974) Class inclusion failure : Cognitive deficit or misleading reference, Child Development, 45, 1122-1125.

Karmiloff-Smith A - (1992) Beyond modularity : A developmental perspective of cognitive science, Cambridge (MA), MIT Press.

Karmiloff-Smith A. - (1994) Precis of beyond modularity : A developmental perspective on cognitive science, Behavioral and Brain Sciences, 17, 693745.

Kipp Harnishfeger K. - (1995) The development of cognitive inhibition. Theories, definitions, and research evidence, in F. N. Dempster et C. J. Brainerd (Edit.), Interference and inhibition in cognition, New York, Academic Press, 176-204.

Kipp Harnishfeger K., Bjorklund D. - (1994) A developmental perspective on individual differences in inhibition, Learning and Individual Differences, 6 , 3.31-3.5.5.

Kohnstamm G. A. - (1963) An evaluation of part of Piaget's theory, Acta Psychologica, 21, 313-356.

Kurtz R. J. - (1978) The effects of substage level of classificatory logic and mode of presentation on consistency and success of class inclusion performance, Dissertation Abstracts International, 38, 6196-6197.

Iane M. K., Hodkin B. - (1985) Role of atypical exemplars of social and nonsocial superordinate categories within the class inclusion paradigm, Developmenal P'sychology, 21, 909-915.

Majeres 1. M., O'Toole J. - (1980) The effect of type of superordinate class and size of array on class-inclusion performance, The Journal of Genetic Psychology, 1.37, 257-265. 
Marantz B. - (1976) Effect of cue salience, cue dimension and dimension preference on class inclusion ability in young children, Dissertation Abstracts International, 36, 7955-7956.

Markman E. M. - (1978) Empirical versus logical solutions to part-whole comparison problems concerning classes and collections, Child Development, 49, 168-177.

Markman E., Seibert J. - (1976) Classes and collections : Internal organization and resulting holistic properties, Cognitive Psychology, 8, 561-577.

McGarrigle J., Grieve R., Hugues M. - (1978) Interpreting inclusion : A contribution to the study of the child's cognitive and linguistic development, Journal of Experimental Child Psychology, 26, 528-550.

Montangero J. - (1991) A constructivist framework for understanding early and late-developing psychological competencies, in $M$. Chandler et M. Chapman (Edit.), Criteria for competence, Hillsdale (N.J), Erlbaum, 111 150.

Moshman D. - (1990) The development of metalogical understanding, in W. F. Overton (Edit.), Reasoning, necessity, and logic : Developmental perspectives, Hillsdale (N.J), Erlbaum, 183-203.

Moshman D., Timmons M. - (1982) The construction of logical necessity, Human Development, 25, 309-323.

Müller U., Sokol B., Overton W. F. - (1999) Developmental sequences in class reasoning and propositional reasoning. Journal of Experimental Child Psychology, 74, 69-106.

Pascual-Leone J. - (1988) Organismic processes for neo-piagetain theories : A dialectical causal account of cognitive development, in A. Demetriou (Edit), The neo-piagetian theories of cognitive development : Toward an integration, Amsterdam, North-Holland, 25-64.

Pascual-Leone J., Bovet M.-C. - (1966) L'apprentissage de la quantification de l'inclusion et la théorie opératoire, Acta Psychologica, 25, 334-356.

Piaget J. - (1977) Recherches sur l'abstraction réfléchissante, Paris, PUF.

Piaget J., Garcia, R. - (1987) Vers une logique des significations, Genève, Murionde.

Piaget J., Inhelder B. - (1967) La genèse des structures logiques élémentaires, $2^{e}$ éd., Neuchâtel, Delachaux \& Niestlé.

Piaget J., Szeminska A. - (1967) La genèse du nombre chez l'enfant, 4* éd., Neuchâtel, Delachaux \& Niestlé.

Politzer G. - (1993) La psychologie du raisonnement : lois de la pragmatique et logique formelle, thèse de doctorat, Université de Paris VIII, Paris.

Russel J. - (2000) Développement cognitif et fonctions exécutives. «L'essentiel de Piaget », in O. Houdé et C. Meljac (Edit.), L'esprit piagetien. Hommage international d̀ Jean Piaget, Paris, Pur, 149-189.

Schröder E., Edelstein W. - (1991) Intrinsic and external constraints on the development of cognitive competence, in $M$. Chandler et $M$. Chapman (Edit.), Criteria for competence, Hillsdale (NJ), Erlbaum, 131 - 150.

Schwebel A. I., Schwebel C. R. - (1974) The relationship between performance on piagetian tasks and impulsive responding, Journal for Research in Mathematics Education, 5, 98-104.

Shipley E. - (1979) The class-inclusion task : Question form and distributive comparisons, Journal of Psycholinguistic Research, 8, 301-331.

Siegel L. S., McCabe A. E., Brand J., Matthews J. - (1978) Evidence for the understanding of class inclusion in preschool children : Linguistic factors and training effects, Child Development, 49, 688-693. 
Sophian C. - (1997) Beyond performance : The signicance of performance for conceptual development, Cognitive Development, 12, 281-303.

Tatarsky J. H. - (1974) The influence of dimensional manipulations on classinclusion performance, Child Development, 45, $1173-1175$.

Thomas H., Horton J. J. - (1997) Competency criteria and the class inclusion task : Modeling judgments and justifications, Developmental Psychology, 33, $1060-1073$.

Trabasso T., Isen A. M., Dolecki P., McLanahan A. G., Riley C. A., Tucker T. - (1978) How do children solve class-inclusion problems?, in R. S. Siegler (Edit.), Children's thinking : What develops?, Hillsdale (N.J), Erlbaum.

Visser M., Das-Smaal F., Kwakman H. - (1996) Impulsivity and negative priming : Evidence for diminished cognitive inhibition in impulsive children, British Journal of Psychology, 87, 131-140.

Voelin C. - (1976) Deux expériences à propos de l'extension dans l'épreuve de la quantification de l'inclusion, Revue suisse de psychologie, 35, 269-284.

Wilkinson A. - (1976) Counting strategies and semantic analysis as applied to class inclusion, Cognitive Psychology, 8, 64-85.

Winer, G. A. - (1978) Enhancement of class inclusion reasoning through verbal context, Journal of Genetic Psychology, 1.32, 299-306.

Winer G. A. - (1980) Class-inclusion reasoning in children : A review of the empirical literature, Child Development, 51, 309-328.

Winer G. A., Falkner R. A. - (1984) The effects of linguistic factors on class inclusion performance in adults and children, The Journal of Genetic Psychology, 145, 251-265.

Wohlwill J. F. - (1968) Responses to class-inclusion questions for verbally and pictorially presented items, Child Development, 39, 449-465.

Youssef Z. I., Guardo C. J. - (1972) The additive composition of classes: The role of perceptual cues, The Journal of Genetic Psychology, 121, 197-205.

(Accepté le 21 janvier 2002.) 